\title{
Análisis de usabilidad web a través de métricas estandarizadas y su aplicación práctica en la plataforma SAEFI
}

\section{Web usability analysis through standardized metrics and its practical application on the SAEFI platform}

\author{
MEX-ALVAREZ, Diana Concepción†*, HERNÁNDEZ-CRUZ, Luz María, UC-RIOS, Carlos Eduardo \\ y CAB-CHAN, José Ramón
}

Universidad Autónoma de Campeche, Facultad de Ingeniería

ID $1^{\mathrm{er}}$ Autor: Diana Concepción, Mex-Alvarez / ORC ID: 0000-0001-9419-7868, Researcher ID Thomson: I-4164-2018, CVU CONACYT ID: 842039

ID $1^{\text {er }}$ Coautor: Luz María, Hernández-Cruz / ORC ID: 0000-0002-0469-5298, Researcher ID Thomson: H-3153-2018, arXiv Author ID: 2234586, CVU CONACYT ID: 662220

ID $2^{\text {do }}$ Coautor: Carlos Eduardo, Uc-Rios / ORC ID: 0000-0003-1321-019X, Researcher ID Thomson: K-6624-2018, CVU CONACYT ID: 88147

ID $3^{\text {er }}$ Coautor: José Ramón, Cab-Chan / ORC ID: 0000-0003-1043-629, Researcher ID Thomson: I-5425-2018, CVU CONACYT ID: 204250

DOI: $10.35429 / J O C T .2019 .9 .3 .15 .24$

Recibido 16 de Enero, 2019; Aceptado 04 Marzo, 2019

\begin{abstract}
Resumen
Hoy en día, todas las Universidades e Instituciones Educativas buscan alcanzar la calidad en sus servicios. En este trabajo se analiza el desempeño de una aplicación web de gestión escolar, tomando en cuenta "la calidad del producto y la calidad en uso", según la norma ISO 25010:2011, con la finalidad de contar con los datos para mejorar u optimizar el sistema. Se propone una metodología para la evaluación de la usabilidad, aplicando dos métodos de evaluación: el método empírico contempla el diseño y aplicación de un instrumento de recogida de datos que evalúa indicadores y métricas por parte de los usuarios de la aplicación web; y el método heurístico discrimina y aplica herramientas de software de uso específico que evalúa automáticamente la aplicación web. La evaluación de usabilidad en su primera fase en el método empírico sobre el modelo de calidad de producto nos arroja una tendencia lineal entre el $60 \%$ y el $80 \%$; en la segunda fase en el método heurístico sobre la calidad de uso respecto a la accesibilidad obtuvo valores de cumplimento entre el $32 \%$ y el $93 \%$, y en velocidad del $71 \%$ al $99 \%$.
\end{abstract}

Usabilidad, Accesibilidad, SAEFI

\begin{abstract}
Today, all Universities and Educational Institutions seek to achieve quality in their services. In this project, it analyzes the performance of a school management web application, taking into account "the quality of the product and the quality in use", according to ISO 25010: 2011, in order to have the data to improve or optimize the system. A methodology for the evaluation of usability is proposed, applying two evaluation methods: the empirical method contemplates the design and application of a data collection instrument that evaluates indicators and metrics by users of the web application; and the heuristic method that discriminates and applies specific use software tools that automatically evaluates the web application. The usability evaluation in its first phase in the empirical method on the product quality model gives us a linear trend between $60 \%$ and $80 \%$; in the second phase about the heuristic method on the quality of use with respect to accessibility, it obtained compliance values between $32 \%$ and $93 \%$, and in speed from $71 \%$ to $99 \%$.
\end{abstract}

Usabilidad, Accesibilidad, SAEFI

Citación: MEX-ALVAREZ, Diana Concepción, HERNÁNDEZ-CRUZ, Luz María, UC-RIOS, Carlos Eduardo y CABCHAN, José Ramón. Análisis de usabilidad web a través de métricas estandarizadas y su aplicación práctica en la plataforma SAEFI. Revista de Tecnologías Computacionales. 2019 3-9: 15-24

\footnotetext{
* Correspondencia del Autor (Correo electrónico: diancmex@uacam.mx)

$\dagger$ Investigador contribuyendo como primer autor.
} 


\section{Introducción}

Hoy en día, todas las Universidades e Instituciones Educativas buscan alcanzar la calidad en sus servicios. Con ello, es indispensable el uso de sistemas de información que permitan automatizar sus procesos y hacerlos más eficientes. (Xool Clavel, Buenfil Paredes, \& Dzul Canche, 2018)

La coordinación de Posgrado de la Facultad de Ingeniería de la Universidad Autónoma de Campeche, ha realizado diferentes mecanismos para la gestión administrativa y académica de los alumnos de posgrado. Sin embargo, con el incremento de matrícula y de la información, surge la necesidad de implementar un sistema de información flexible, amigable y eficiente.

Tomando en cuenta que en las universidades debemos desarrollar en nuestros estudiantes habilidades para crear productos de software de calidad a nivel internacional. (Barrios García, Sahagun Montoya, Bañuelos Rodarte, \& Moreira Galvan, 2018), se desarrolló un "Sistema de Administración Escolar de la Facultad de Ingeniería" (SAEFI), adecuado para la gestión administrativa y académica de los alumnos de posgrado de esta Universidad, en el que participaron estudiantes y maestros(Mex Alvarez, Escamilla de la Cruz, Estrada Segovia, Hernández Cruz, \& Ortiz Cuevas, 2019).

El objetivo del presente trabajo es evaluar usabilidad de la aplicación web SAEFI y determinar la pertinencia de su actualización visual y funcional (Front-end) considerando métricas estandarizadas. Lo anterior con la finalidad de poder usar los resultados para la mejorar del sistema actual.

La usabilidad es la cualidad de una página web o programa informático de ser de fácil acceso, legible, descarga rápida de información, contar con funciones y menús entendibles, por los usuarios pueden realizar tareas específicas fáciles y en menos tiempo. (Mascheroni, Greiner, Petris, Dapozo, \& Estayno, 2012).

Esta investigación aporta como valor agregado, el uso aplicativo de la Norma Estándar Internacional ISO 25010:2011 para la evaluación de la usabilidad de un producto de software.
El resto del artículo está organizado de la siguiente manera: En la sección 2 se definen los conceptos teóricos científicos de Usabilidad y de la Norma Estándar Internacional ISO 25010:2011; en la sección 3, se establecen los pasos a seguir en el estudio de la investigación; en la sección 4 se evalúa la calidad de uso de la página web; en la sección 5 se evalúa la calidad de producto en la página web; posteriormente se presentan los resultados en la sección 6 y por último las conclusiones en la sección 7.

\section{Marco Teórico}

La Usabilidad se define como "el grado en que un producto o sistema puede ser utilizado por usuarios específicos para lograr objetivos específicos con eficacia, eficiencia y satisfacción en un contexto específico de uso". (Enriquez \& Casas, 2013)

La evaluación de la usabilidad puede ser de forma heurística o empírica. Los métodos heurísticos o no empíricos implican la participación de expertos en usabilidad que realizan el proceso de evaluación. Por otra parte, los métodos empíricos constan de técnicas e instrumentos que requieren de la participación de los usuarios. (Rubin \& Dana, 2008)

A través de la norma estándar internacional ISO 25010:2011 se evaluará de la usabilidad de la aplicación web SAEFI, aplicando métodos empíricos y heurísticos.

El método empírico contempla el diseño y aplicación de un instrumento de recogida de datos que evalúa indicadores y métricas por parte de los usuarios de la aplicación web SAEFI.

El método heurístico discrimina y aplica herramientas de software de uso específico que evalúa automáticamente la aplicación web SAEFI.

\section{La Norma ISO 25010 define:}

i. Un modelo de calidad del producto compuesto por ocho características que se relacionan con las propiedades estáticas del software y las propiedades dinámicas de la computadora sistema. El modelo es aplicable tanto a los sistemas informáticos como a los productos de software. 
ii. Un modelo de calidad en uso compuesto por cinco características que se relacionan con el resultado de la interacción cuando un producto se utiliza en un contexto particular de uso. Este modelo de sistema es aplicable a todo sistema humano-computadora, incluyendo tanto sistemas informáticos y productos de software en uso. (British Standards Institution, 2011)

La Figura 1 muestra la estructura principal de la norma estándar internacional ISO 25010, resaltando los Modelos y apartados considerados de interés en el presente artículo.

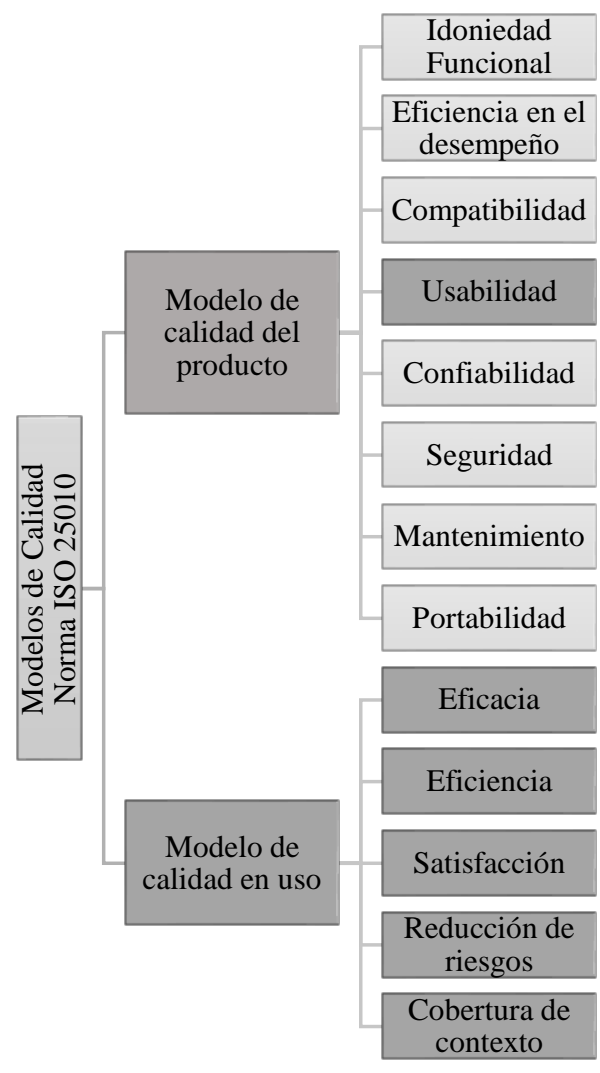

Figura 1 Modelos de Calidad de la Norma ISO 25010 Fuente: Elaboración Propia

Claramente se observan dos enfoques para evaluar la usabilidad de un producto de software o sistema de información con base en la norma ISO 25010, como parte de un modelo de calidad del producto y con un modelo de calidad de uso.

\section{Método de evaluación}

Debido a que el sistema ya se encuentra operando, se propone una metodología centrada en usuarios finales con el fin de reformular sus requerimientos y así lograr su rediseño. (Jiménez \& Lugones, 2015)
La Norma ISO 25010 contempla a la usabilidad bajo dos puntos de vista distintos: uno que contempla a la usabilidad desde el punto de vista del software, como producto en sí mismo; y el otro punto de vista desde la usabilidad de uso, desde la perspectiva del usuario. (Arroyo Paz, 2019)

En base a esto, se analizan los dos enfoques de interés:

a) Evaluación de la aplicación web SAEFI como parte de un Modelo de calidad del producto usando un método empírico con un instrumento para la recogida de datos para la evaluación por parte de los usuarios. Las fases de este enfoque son:

- Construcción del instrumento,

- $\quad$ Aplicación del instrumento.

- Resultados

b) Evaluación de la aplicación web SAEFI como parte de un Modelo de calidad de uso empleando un método heurístico con la aplicación de herramientas de software de uso específico para el análisis y evaluación de forma automática. Las fases de este enfoque son:

- Herramientas de software de uso específico, y

- $\quad$ Aplicación de las Herramientas.

- Resultados.

La Figura 2 muestra las Fases de estudio en los Métodos empírico y heurísticos para evaluar la aplicación web SAEFI con la Norma Estándar Internacional ISO 25010.
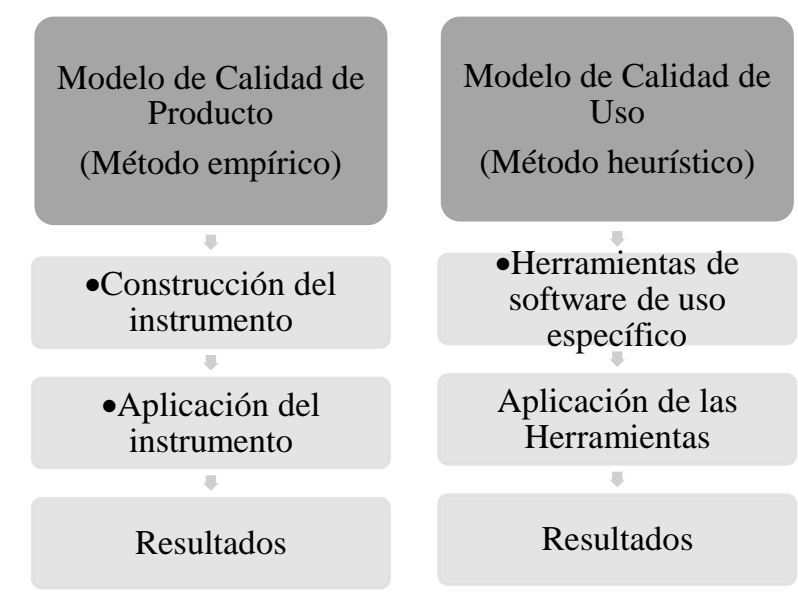

Figura 2 Fases de estudio para evaluar la aplicación web SAEFI con la norma ISO 25010

Fuente: Elaboración Propia

MEX-ALVAREZ, Diana Concepción, HERNÁNDEZ-CRUZ, Luz María, UC-RIOS, Carlos Eduardo y CAB-CHAN, José Ramón. Análisis de usabilidad web a través de métricas estandarizadas y su aplicación práctica en la plataforma SAEFI. Revista de Tecnologías Computacionales. 2019 


\section{Evaluación de la aplicación web SAEFI como parte de un Modelo de calidad del producto}

\section{Construcción del instrumento}

El modelo de calidad del producto, de la norma estándar internacional ISO 25010, clasifica las propiedades de calidad del producto del software o sistemas de información en ocho características: idoneidad funcional, eficiencia de rendimiento, compatibilidad, facilidad de uso, fiabilidad, seguridad, mantenibilidad $y$ portabilidad.

Específicamente la característica de Facilidad de uso o Usabilidad está compuesta de seis subcaracterísticas: adecuación reconocible, capacidad de aprendizaje, operabilidad, protección contra errores, estética de la interfaz de usuario y accesibilidad. Es importante mencionar que dichas características están relacionadas entre sí. (Febrero, Calero, \& Moraga, 2016)

La Figura 3 muestra las subcaracterísticas a evaluar de la Usabilidad como parte del Modelo de calidad de producto de la Norma ISO 25010 en la aplicación web SAEFI.

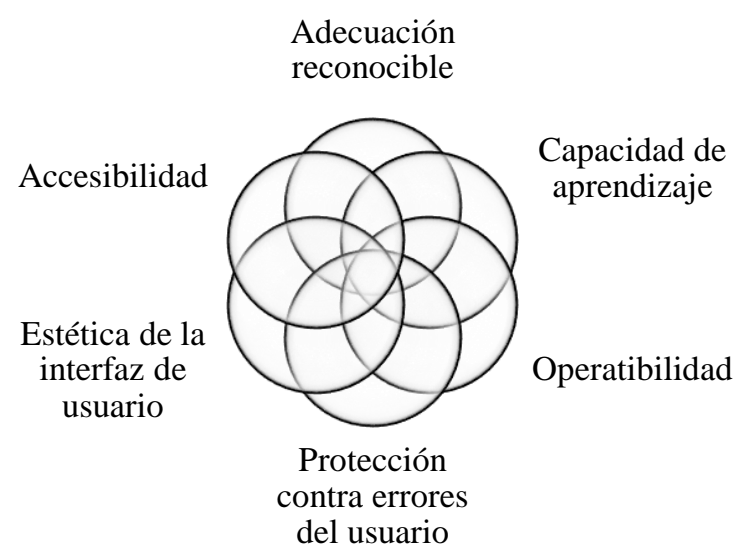

Figura 3 Subcaracterísticas de Usabilidad en el Modelo de calidad del producto, Norma ISO 25010

Fuente: Elaboración Propia

El desarrollo del instrumento de recogida de datos para la evaluación de la Usabilidad (facilidad de uso) de la aplicación web SAEFI, se diseña fijando cuatro secciones principales: capacidad para la identificación apropiada, accesibilidad, operabilidad y estética de la interfaz. La capacidad para la identificación apropiada busca evaluar la comprensión del cómo se llevan a cabo las funciones de la aplicación web SAEFI.
La accesibilidad es la encargada de evaluar si las funciones de la aplicación SAEFI son fáciles de llevar a cabo por el usuario. La operabilidad permite evaluar cómo los usuarios operan la aplicación web SAEFI, si es sencillo corregir errores cometidos por ellos mismos. La estética de la interfaz concede evaluar si a los usuarios les agrada de manera visual la aplicación SAEFI. La Tabla 1 muestra las subcacterísticas de la característica Usabilidad de la Norma ISO 25010 con su indicador de evaluación y la sección del instrumento de recogida de datos con el que se relaciona. (França \& Soares, 2015)

\begin{tabular}{|c|c|c|}
\hline $\begin{array}{c}\text { Subcategoría } \\
\text { de Calidad del } \\
\text { Producto para } \\
\text { la } \\
\text { característica } \\
\text { de Usabilidad }\end{array}$ & $\begin{array}{c}\text { Indicador de } \\
\text { Subcaracterística }\end{array}$ & $\begin{array}{c}\text { Sección del } \\
\text { Instrumento de } \\
\text { recogida de datos } \\
\text { (Número de } \\
\text { preguntas } \\
\text { asociadas al } \\
\text { indicador / Total } \\
\text { de preguntas) }\end{array}$ \\
\hline $\begin{array}{l}\text { Adecuación } \\
\text { Reconocible }\end{array}$ & $\begin{array}{l}\text { El grado en que los usuarios } \\
\text { pueden reconocer si } \\
\text { producto o sistema } \\
\text { adecuado } \\
\text { necesidades. }\end{array}$ & $\begin{array}{l}\text { Capacidad para } \\
\text { la identificación } \\
\text { apropiada } \\
(3 / 15)\end{array}$ \\
\hline $\begin{array}{l}\text { Capacidad de } \\
\text { aprendizaje }\end{array}$ & $\begin{array}{l}\text { El grado en que un producto } \\
\text { o sistema puede ser utilizado } \\
\text { por usuarios específicos } \\
\text { para lograr objetivos } \\
\text { específicos de aprendizaje } \\
\text { para utilizar el producto o } \\
\text { sistema con efectividad, } \\
\text { eficiencia, libertad de riesgo } \\
\text { y satisfacción en un } \\
\text { determinado } \\
\text { contexto de uso }\end{array}$ & $\begin{array}{l}\text { Capacidad para } \\
\text { la identificación } \\
\text { apropiada }(3 / 15)\end{array}$ \\
\hline Operabilidad & $\begin{array}{l}\text { El grado en que un producto } \\
\text { o sistema tiene atributos que } \\
\text { lo hacen fácil de operar y } \\
\text { controlar. }\end{array}$ & $\begin{array}{l}\text { Operabilidad } \\
(3 / 15)\end{array}$ \\
\hline $\begin{array}{l}\text { Protección } \\
\text { contra errores } \\
\text { del usuario } \\
\end{array}$ & $\begin{array}{l}\text { Grado en que un sistema } \\
\text { protege a los usuarios contra } \\
\text { errores. }\end{array}$ & $\begin{array}{l}\text { Capacidad para } \\
\text { la identificación } \\
\text { apropiada }(1 / 15) \\
\end{array}$ \\
\hline $\begin{array}{l}\text { Estética de la } \\
\text { interfaz de } \\
\text { usuario }\end{array}$ & $\begin{array}{l}\text { El grado en que una interfaz } \\
\text { de usuario permite una } \\
\text { interacción agradable y } \\
\text { satisfactoria para el usuario. }\end{array}$ & $\begin{array}{l}\text { Estética de la } \\
\text { interfaz }(2 / 15)\end{array}$ \\
\hline Accesibilidad & $\begin{array}{l}\text { El grado en que un producto } \\
\text { o sistema puede ser utilizado } \\
\text { por personas con la más } \\
\text { amplia gama de } \\
\text { características } \\
\text { Capacidades para lograr un } \\
\text { objetivo específico en un } \\
\text { contexto específico de uso. }\end{array}$ & $\begin{array}{l}\text { Accesibilidad } \\
(3 / 15)\end{array}$ \\
\hline
\end{tabular}

Tabla 1 Relación de subcaracterística/indicador y sección del instrumento para evaluar la Usabilidad en la aplicación web SAEFI

El instrumento de recogida de datos consta de un total de quince preguntas de opción múltiple, para conservar la atención y objetividad de los usuarios al responder el cuestionario. Cada sección del cuestionario tiene un conjunto de preguntas diseñadas para que los usuarios respondan de acuerdo con su experiencia al usar la aplicación web SAEFI. 
La Tabla 2 muestra la relación de cada subcaracterística de Usabilidad definidas en la norma ISO 25010 con las preguntas del instrumento de recogida de datos y las métricas provistas para la evaluación.

\begin{tabular}{|c|c|c|}
\hline $\begin{array}{l}\text { Subcaracterístic } \\
\text { as de calidad del } \\
\text { producto para } \\
\text { la característica } \\
\text { de Usabilidad } \\
\text { (Indicadores } \\
\text { establecidos) }\end{array}$ & $\begin{array}{l}\text { Pregunta en el } \\
\text { instrumento }\end{array}$ & $\begin{array}{l}\text { Métricas en el } \\
\text { Instrumento }\end{array}$ \\
\hline $\begin{array}{l}\text { Adecuación } \\
\text { reconocible } \\
\mathbf{I}_{\mathrm{AR} 1}=\text { Pregunta } 1 \\
\mathbf{I}_{\mathrm{AR} 2}=\text { Pregunta } 2 \\
\mathbf{I}_{\mathrm{AR} 3}=\text { Pregunta } 3\end{array}$ & $\begin{array}{l}\text { 1. ¿Las funciones del } \\
\text { Portal de Posgrado son } \\
\text { claras y sencillas? } \\
\text { 2. ¿Cómo considera el } \\
\text { acceso a las funciones } \\
\text { del Portal de Posgrado? } \\
\text { 3. ¿Considera necesario } \\
\text { agregar una sección de } \\
\text { ayuda en el Portal de } \\
\text { Posgrado? (En caso de } \\
\text { ser "si", justifique su } \\
\text { respuesta en el apartado } \\
\text { "Otro"). } \\
\end{array}$ & $\begin{array}{l}\text { Totalmente de acuerdo/De } \\
\text { acuerdo/Ni acuerdo ni en } \\
\text { desacuerdo/En } \\
\text { desacuerdo/Totalmente en } \\
\text { desacuerdo } \\
\text { Muy fácil/Fácil/Ni fácil ni } \\
\text { difícil/Difícil/Muy difícil } \\
\text { Sí/Otro }\end{array}$ \\
\hline $\begin{array}{l}\text { Capacidad de } \\
\text { aprendizaje } \\
\text { ICA1 =Pregunta } 4 \\
\text { I }_{\text {CA2 }}=\text { Pregunta } 5 \\
\text { ICA3 }_{\text {=Pregunta } 6}\end{array}$ & $\begin{array}{l}\text { 4. ¿Logra completar tareas } \\
\text { específicas de manera } \\
\text { simple? (En caso de no } \\
\text { ser así, justifique su } \\
\text { respuesta en el apartado } \\
\text { "Otro"). } \\
\text { 5. ¿Cómo considera que se } \\
\text { encuentran localizadas } \\
\text { las secciones y } \\
\text { funciones del Portal de } \\
\text { Posgrado? } \\
\text { 6. ¿Entiende de manera } \\
\text { clara las funciones que } \\
\text { ofrece el Portal de } \\
\text { Posgrado? }\end{array}$ & 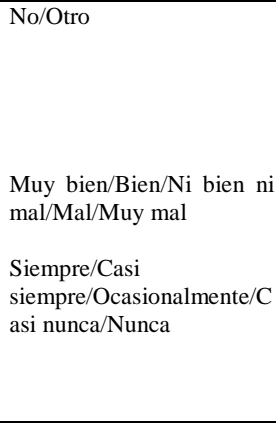 \\
\hline $\begin{array}{l}\text { Operabilidad } \\
\text { Io1 }=\text { Pregunta } 7 \\
\text { I02 }=\text { Pregunta } 8 \\
\text { I03 =Pregunta } 9\end{array}$ & $\begin{array}{l}\text { 7. ¿Al utilizar el Portal de } \\
\text { Posgrado se presenta } \\
\text { algún tipo de error? } \\
\text { (Errores generados por } \\
\text { el sistema) } \\
\text { 8. ¿Considera que el Portal } \\
\text { de Posgrado cuenta con } \\
\text { un mecanismo de } \\
\text { mensajes o alertas } \\
\text { apropiado? } \\
\text { 9. ¿Puede recordar con } \\
\text { facilidad los mensajes o } \\
\text { alertas del Portal de } \\
\text { Posgrado que considere } \\
\text { importante? }\end{array}$ & $\begin{array}{l}\text { Siempre/Casi } \\
\text { siempre/Ocasionalmente/C } \\
\text { asi nunca/Nunca } \\
\text { Siempre/Casi } \\
\text { siempre/Ocasionalmente/C } \\
\text { asi nunca/Nunca }\end{array}$ \\
\hline $\begin{array}{l}\text { Protección } \\
\text { contra errores } \\
\text { del usuario } \\
\text { IPEI=Pregunta } \\
\text { 10 }\end{array}$ & $\begin{array}{l}10 . \\
\text { conocimiento sobre qué } \\
\text { tipo de datos debe } \\
\text { ingresar en cada } \\
\text { apartado del Portal de } \\
\text { Posgrado? }\end{array}$ & $\begin{array}{l}\text { Siempre/Casi } \\
\text { siempre/Ocasionalmente/C } \\
\text { asi nunca/ Nunca }\end{array}$ \\
\hline $\begin{array}{l}\text { Estética de la } \\
\text { interfaz } \\
\text { I }_{\text {EI1 }}=\text { Pregunta } 11 \\
\text { I }_{\mathrm{EL} 2}=\text { Pregunta } 12\end{array}$ & $\begin{array}{l}11 . \\
\text { visualmente agradable } \\
\text { utilizar el Portal de } \\
\text { Posgrado? } \\
\text { 12. ¿Recomenda } \\
\text { ría el uso del Portal de } \\
\text { Posgrado? } \\
\end{array}$ & Sí/No \\
\hline $\begin{array}{l}\text { Accesibilidad } \\
\mathbf{I}_{\mathrm{A} 1}=\text { Pregunta } 13 \\
\mathbf{I}_{\mathrm{A} 2}=\text { Pregunta } 14 \\
\mathbf{I}_{\mathrm{A} 3}=\text { Pregunta } 15\end{array}$ & $\begin{array}{l}13 . \text { ¿Le toma } \\
\text { demasiado tiempo } \\
\text { realizar alguna actividad } \\
\text { en el Portal de } \\
\text { Posgrado? } \\
14 . \quad \text { ¿Con qué } \\
\text { frecuencia confunde los } \\
\text { botones o etiquetas del } \\
\text { Portal de Posgrado? } \\
15 . \quad \text { ¿Es } \\
\text { complicado leer los } \\
\text { elementos escritos en la } \\
\text { plataforma con los } \\
\text { tamaños elegidos para el } \\
\text { texto? }\end{array}$ & $\begin{array}{l}\text { Siempre/Casi } \\
\text { siempre/Ocasionalmente/C } \\
\text { asi nunca/Nunca } \\
\text { Siempre/Casi } \\
\text { siempre/Ocasionalmente/C } \\
\text { asi nunca/Nunca } \\
\text { Siempre/Casi } \\
\text { siempre/Ocasionalmente/C } \\
\text { asi nunca/Nunca }\end{array}$ \\
\hline
\end{tabular}

Tabla 2 Relación de subcaracterísticas de Usabilidad de la norma ISO 25040 con preguntas y métricas en el instrumento de recogida de datos. Definiendo los indicadores a evaluar.
La herramienta utilizada para elaborar el instrumento de recogida de datos fue Google Forms de la compañía Google permitiendo su creación de manera fácil y formal, con una interfaz intuitiva y amigable. Además, los datos obtenidos se registran de manera automática en Hojas de cálculo de Google y brinda la facilidad de enviar por correo electrónico o compartir el vínculo del cuestionario para que solo los usuarios deseados puedan resolverlo.

\section{Aplicación del instrumento}

El universo de muestreo para la investigación. Fueron los 17 docentes adscritos a la Universidad Autónoma de Campeche impartieron cátedra en los 3 programas educativos ofrecidos: Maestría en Ingeniería con Orientación en Energía, Maestría en Ingeniería con Orientación en Vías Terrestres y la Maestría en Ciencias de la Preservación de Materiales, en el ciclo escolar 2018-2019 FII. El instrumento de recogida de datos fue enviado vía correo electrónico institucional a todos los elementos del muestreo, de los 17 elementos del universo, 11 docentes contestaron el instrumento de recogida de datos, lo que significa el $64 \%$. Todos los resultados aquí mostrados consideran a estos 11 elementos el $100 \%$ del estudio.

\section{Evaluación de la aplicación web SAEFI como parte de un Modelo de calidad de uso, norma estándar internacional ISO 25010}

El método heurístico permite evaluar sin intervención de los usuarios. Con este enfoque, la aplicación web SAEFI se somete a la evaluación automática de seis herramientas de software de uso específico:

GTmetrix es una herramienta para evaluar el rendimiento de una aplicación web, analizando la velocidad de carga de la página. $\mathrm{Al}$ finalizar el análisis GTmetrix genera un informe con el porcentaje de velocidad alcanzado. (GT.net, 2019)

Google PageSpeed Insights analiza el rendimiento de front-end de su sitio y ofrece sugerencias de optimización. Proporcionando a su sitio web una puntuación entre 0 y 100 puntos. Una puntuación de 85 o superior indica una página con buen rendimiento, esta herramienta puede brindarle una gran información sobre el rendimiento de su sitio web. (Google, 2018) 
Uptrends valora la velocidad de sitios web con una puntuación entre 0 y 100 puntos, como información adicional, proporciona recomendaciones de optimización detalladas. (UPTRENDS, 2019)

Lighthouse. Es una herramienta de Chrome, se ejecuta como una extensión o desde la línea de comandos, proporcionándole la URL para auditar. Lighthouse ejecuta una serie de pruebas, generando un informe del porcentaje alcanzado por la página sobre la velocidad, la accesibilidad, buenas prácticas y optimización en los motores de búsqueda. (Google, 2019)

Examinator. - "Es un servicio en línea para evaluar de modo automático la accesibilidad de una página web, usando como referencia algunas técnicas recomendadas por las Pautas de Accesibilidad para el Contenido Web 2.0 (WCAG 2.0)". (Benavidez, 2015). (Fundación del Seminario Iberoamericano sobre Discapacidad y Accesibilidad en la Red, 2009) Examinator proporciona un informe detallado de las pruebas considerando prorcionando una calificación global del 1 al 10

Functional Accessibility Evaluator 2.0. El Evaluador de Accesibilidad Funcional (FAE) evalúa un sitio web o una sola página web según los requisitos de Nivel A y AA, (los niveles más altos de tres) de las Pautas de Accesibilidad para el Contenido Web (WCAG) 2.0 del W3C.

Pautas de Accesibilidad para el Contenido Web (WCAG) 2.0 del W3C. (University of Illinois, 2019) La Tabla 4 muestra las propiedades a evaluar relacionadas con cada una de las características de Usabilidad en el Modelo de calidad de uso en la norma estándar internacional ISO 25010 y la herramienta de software utilizada.

\begin{tabular}{|l|l|l|}
\hline $\begin{array}{c}\text { Propiedad de } \\
\text { la aplicación } \\
\text { web SAEFI a } \\
\text { evaluar }\end{array}$ & $\begin{array}{c}\text { Características } \\
\text { asociadas a la } \\
\text { Usabilidad en el } \\
\text { Modelo de calidad } \\
\text { de uso norma ISO }\end{array}$ & $\begin{array}{c}\text { Herramienta de } \\
\text { 25010 } \\
\text { específico para evaluar } \\
\text { una aplicación web }\end{array}$ \\
\hline Velocidad & $\begin{array}{l}\text { Eficiencia y } \\
\text { eficacia }\end{array}$ & $\begin{array}{l}\text { GTmetrix } \\
\text { PageSpeed Insigths } \\
\text { Uptrends } \\
\text { Lighthouse }\end{array}$ \\
\hline Accesibilidad & Cobertura del & $\begin{array}{l}\text { Examinator } \\
\text { Functional } \\
\text { contexto }\end{array}$ \\
& & $\begin{array}{l}\text { Accessibility } \\
\text { Evaluator } 2.0 \\
\text { Lighthouse }\end{array}$ \\
\hline
\end{tabular}

Tabla 3 Herramientas de software asociadas a la Evaluación automática de las características de Usabilidad en el Modelo de calidad de uso de la norma ISO 25010.

\section{Resultados}

Los resultados de la evaluación como parte del modelo de calidad de producto, empleando el método empírico se obtuvieron descargando la hoja de cálculo que genera Google Forms con los registros obtenidos de la encuesta. Se organizó conforme a las subcaracterísticas de Usabilidad de la norma estándar internacional ISO 25010. La Tabla 3 muestra los resultados obtenidos.

\begin{tabular}{|c|c|c|c|c|}
\hline \multicolumn{5}{|c|}{ Adecuación Reconocible } \\
\hline \multirow{2}{*}{$\begin{array}{l}\text { Métricas } \\
\text { ('métrica } \\
\text { óptima) }\end{array}$} & \multicolumn{3}{|c|}{ Indicadores } & \multirow{2}{*}{$\begin{array}{c}\text { Porcentaje obtenido } \\
\text { por subcaracterística } \\
\text { de Usabilidad }\end{array}$} \\
\hline & $\mathrm{I}_{\mathrm{AR} 1}$ & $\mathrm{I}_{\mathrm{AR} 2}$ & $\mathrm{I}_{\mathrm{AR} 3}$ & \\
\hline $1^{*}$ & $54.5 \%$ & $63.6 \%$ & $90.9 \%$ & $69.7 \%$ \\
\hline 2 & $45.5 \%$ & $36.4 \%$ & $9.1 \%$ & $30.3 \%$ \\
\hline 3 & $0 \%$ & $0 \%$ & & $0 \%$ \\
\hline 4 & $0 \%$ & $0 \%$ & & $0 \%$ \\
\hline 5 & $0 \%$ & $0 \%$ & & $0 \%$ \\
\hline \multicolumn{5}{|c|}{ Capacidad de Aprendizaje } \\
\hline \multirow{2}{*}{$\begin{array}{l}\text { Métricas } \\
\text { ("métrica } \\
\text { óptima) }\end{array}$} & \multicolumn{3}{|c|}{ Indicadores } & \multirow{2}{*}{$\begin{array}{c}\text { Porcentaje obtenido } \\
\text { por subcaracterística } \\
\text { de Usabilidad }\end{array}$} \\
\hline & $\mathrm{I}_{\mathrm{CA} 1}$ & $\mathrm{I}_{\mathrm{CA} 2}$ & $\mathrm{I}_{\mathrm{CA} 3}$ & \\
\hline $1^{*}$ & $100 \%$ & $45.5 \%$ & $63.6 \%$ & $69.7 \%$ \\
\hline 2 & $0 \%$ & $54.5 \%$ & $36.4 \%$ & $30.3 \%$ \\
\hline 3 & & $0 \%$ & $0 \%$ & $0 \%$ \\
\hline 4 & & $0 \%$ & $0 \%$ & $0 \%$ \\
\hline 5 & & $0 \%$ & $0 \%$ & $0 \%$ \\
\hline \multicolumn{5}{|c|}{ Operabilidad } \\
\hline \multirow{2}{*}{$\begin{array}{l}\text { Métricas } \\
\left({ }^{*} \text { métrica }\right. \\
\text { óptima) }\end{array}$} & \multicolumn{3}{|c|}{ Indicadores } & \multirow{2}{*}{$\begin{array}{c}\text { Porcentaje obtenido } \\
\text { por subcaracterística } \\
\text { de Usabilidad }\end{array}$} \\
\hline & Io1 & $\mathrm{I}_{\mathrm{O} 2}$ & $\mathrm{I}_{\mathrm{O} 3}$ & \\
\hline $1^{*}$ & $63.6 \%$ & $36.4 \%$ & $27.3 \%$ & $42.4 \%$ \\
\hline 2 & $27.3 \%$ & $54.5 \%$ & $54.5 \%$ & $45.4 \%$ \\
\hline 3 & $9.1 \%$ & $0 \%$ & $9.1 \%$ & $6.1 \%$ \\
\hline 4 & $0 \%$ & $9.1 \%$ & $9.1 \%$ & $6.1 \%$ \\
\hline 5 & $0 \%$ & $0 \%$ & $0 \%$ & $0 \%$ \\
\hline \multicolumn{5}{|c|}{ Protección contra errores } \\
\hline \multirow{2}{*}{$\begin{array}{l}\text { Métricas } \\
\left({ }^{*} \text { métrica }\right. \\
\text { óptima) }\end{array}$} & \multicolumn{3}{|c|}{ Indicadores } & \multirow{2}{*}{$\begin{array}{c}\text { Porcentaje obtenido } \\
\text { por subcaracterística } \\
\text { de Usabilidad }\end{array}$} \\
\hline & \multicolumn{3}{|c|}{ IEE1 } & \\
\hline $1^{*}$ & \multicolumn{3}{|c|}{$72.2 \%$} & $72.2 \%$ \\
\hline 2 & \multicolumn{3}{|c|}{$27.3 \%$} & $27.3 \%$ \\
\hline 3 & \multicolumn{3}{|c|}{$0 \%$} & $0 \%$ \\
\hline 4 & \multicolumn{3}{|c|}{$0 \%$} & $0 \%$ \\
\hline 5 & \multicolumn{3}{|c|}{$0 \%$} & $0 \%$ \\
\hline \multicolumn{5}{|c|}{ Estética de la interfaz } \\
\hline Métricas & \multicolumn{3}{|c|}{ Indicadores } & Porcentaje obtenido \\
\hline $\begin{array}{l}\text { ('métrica } \\
\text { óptima) }\end{array}$ & IEI1 & & EI2 & $\begin{array}{c}\text { por subcaracterística } \\
\text { de Usabilidad }\end{array}$ \\
\hline $1^{*}$ & $100 \%$ & 100 & & $100 \%$ \\
\hline 2 & $0 \%$ & $0 \%$ & & $0 \%$ \\
\hline & & Acce & bilidad & \\
\hline Métricas & Indicad & & & Porcentaje obtenido \\
\hline $\begin{array}{l}\text { ("métrica } \\
\text { óptima) }\end{array}$ & $\mathrm{I}_{\mathrm{A} 1}$ & $\mathrm{I}_{\mathrm{A} 2}$ & $\mathrm{I}_{\mathrm{A} 3}$ & $\begin{array}{c}\text { por subcaracterística } \\
\text { de Usabilidad }\end{array}$ \\
\hline $1^{*}$ & $45.5 \%$ & $45.5 \%$ & $90.9 \%$ & $60.6 \%$ \\
\hline 2 & $54.5 \%$ & $45.5 \%$ & $9.1 \%$ & $36.4 \%$ \\
\hline 3 & $0 \%$ & $9.0 \%$ & $0 \%$ & $3 \%$ \\
\hline 4 & $0 \%$ & $0 \%$ & $0 \%$ & $0 \%$ \\
\hline 5 & $0 \%$ & $0 \%$ & $0 \%$ & $0 \%$ \\
\hline
\end{tabular}

Tabla 4 Resultados de la evaluación acorde a los indicadores y métricas del instrumento de recogida de datos.

MEX-ALVAREZ, Diana Concepción, HERNÁNDEZ-CRUZ, Luz María, UC-RIOS, Carlos Eduardo y CAB-CHAN, José Ramón. Análisis de usabilidad web a través de métricas estandarizadas y su aplicación práctica en la plataforma SAEFI. Revista de Tecnologías Computacionales. 2019 
En el Gráfico 1, podemos observar cada subcaracterística con los valores alcanzados en la métrica óptima, con una línea de tendencia entre el $60 \%$ y el $80 \%$.

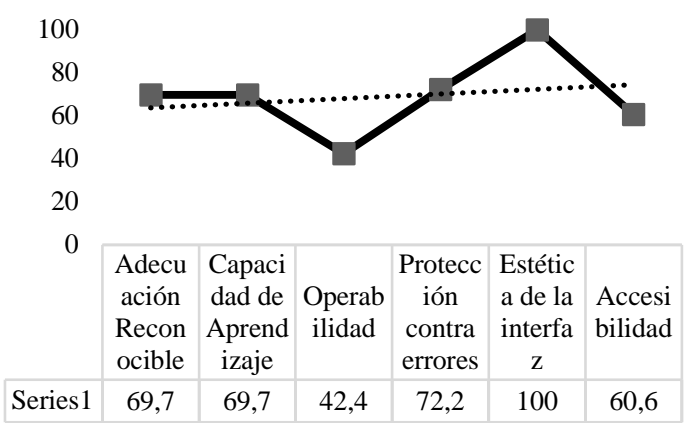

Gráfico 1 Valores alcanzados con métrica óptima por Subcaracterística en el Modelo de calidad del producto.

En seguida, se presenta una síntesis ponderada por cada subcaracterísticas de Usabilidad definidas en el Modelo de calidad de producto de la norma estándar internacional ISO 25010 consolidadas a partir de la métrica óptima (el mejor valor obtenido de satisfacción por parte del usuario) de cada indicador establecido por subcaracterística detallados en la Tabla 2 y exhibiendo el impacto de cada uno de ellos. Al analizar la evaluación correspondiente a la primera subcaracterística denominada Adecuación Reconocible se obtiene un $\mathbf{6 9 . 7 \%}$ de respuestas con métrica óptima, proporcional a los indicadores $\mathrm{I}_{\mathrm{AR} 1}$ un $26 \%$, $\mathrm{I}_{\mathrm{AR} 2}$ un $30 \%$ y $\mathrm{I}_{\mathrm{AR} 3}$ un $44 \%$ de respuestas con métrica óptima. El Gráfico 2 muestra el ponderado porcentual de respuestas con métrica óptima para los tres indicadores de la subcaracterística Adecuación Reconocible.

\section{Adecuación Reconocible}

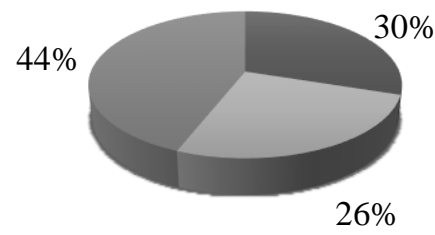

IAR 1 IAR2 IAR3

Gráfico 2 Evaluación de métrica óptima para los indicadores de la subcaracterística Adecuación Reconocible

La siguiente subcaracterística es Capacidad de Aprendizaje, que obtiene un 69.7\% de respuestas con métrica óptima, proporcional a los indicadores $\mathrm{I}_{\mathrm{CA} 1}$ un $48 \%$, ICA2 un $22 \%$ y I $\mathrm{CA3}$ un $30 \%$ de respuestas con métrica óptima.
El Gráfico 3 muestra el ponderado porcentual de respuestas con métrica óptima para los tres indicadores de la subcaracterística Capacidad de Aprendizaje.

Para la subcaracterística Operabilidad se obtiene un $\mathbf{4 2 . 4 \%}$ de respuestas con métrica óptima, proporcional a los indicadores Io1 un $50 \%$ IO2 un $29 \%$ y Io3 un $21 \%$ de respuestas con métrica óptima. La Gráfico 4 muestra el ponderado porcentual de respuestas con métrica óptima para los tres indicadores de la subcaracterística Operabilidad.

\section{Capacidad de Aprendizaje}

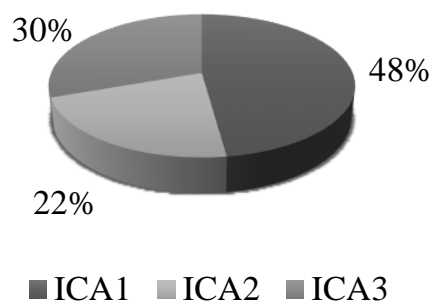

Gráfico 3 Evaluación de métrica óptima para los indicadores de la subcaracterística Capacidad de Aprendizaje

\section{Operabilidad}

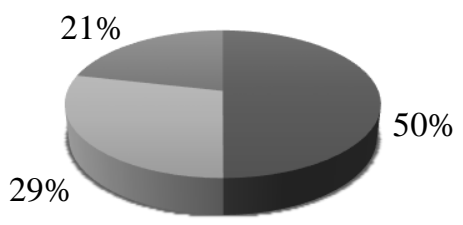

IO1 $\quad \mathrm{IO} 2 \square \mathrm{IO} 3$

Gráfico 4 Evaluación de métrica óptima para los indicadores de la subcategoría Operabilidad.

La subcaracterística Protección contra errores del usuario obtiene para el único indicador evaluado IPE1 el $72.7 \%$ de respuestas con métrica óptima.

La subcaracterística Estética de la interfaz un $100 \%$ de respuestas con métrica óptima, proporcional a los indicadores $\mathrm{I}_{\mathrm{EI} 1}$ un $50 \%$ y el IEI2 un $50 \%$, es decir en ambas preguntas se obtiene el $100 \%$ de respuestas con la métrica óptima.

El Gráfico 5 muestra el ponderado porcentual de respuestas con métrica óptima para los dos indicadores de la subcaracterística Estética de la interfaz. 


\section{Estética de la Interfaz}

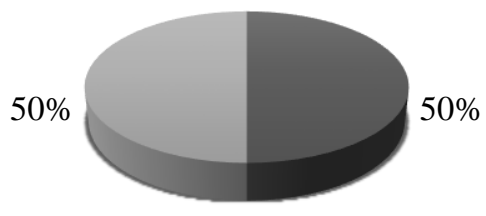

IEI1 IEI2

Gráfico 5 Evaluación de métrica óptima para los indicadores de la subcategoría Estética de la interfaz

Por último, la subcaracterística Accesibilidad se obtiene un $\mathbf{6 0 . 6 \%}$ de respuestas con métrica óptima, proporcional a los indicadores $\mathrm{I}_{\mathrm{A} 1}$ un $25 \%, \mathrm{I}_{\mathrm{A} 2}$ un $25 \%$ y $\mathrm{I}_{\mathrm{A} 3}$ un $50 \%$ de respuestas con la métrica óptima.

El Gráfico 6 muestra el ponderado porcentual de respuestas con métrica óptima para los tres indicadores de la subcaracterística Accesibilidad.

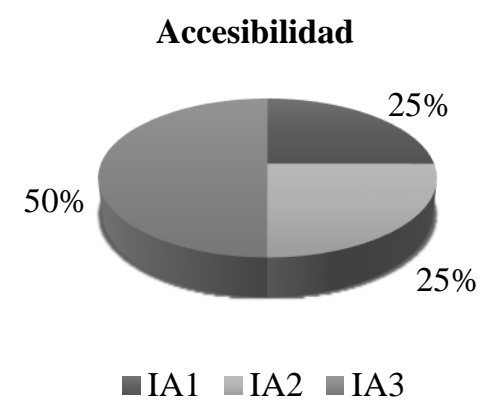

Gráfico 6 Evaluación de métrica óptima para los indicadores de la subcategoría Accesibilidad

Los resultados de la evaluación de la aplicación web SAEFI como parte de un modelo de calidad de uso, norma estándar internacional ISO 25010 empleando el método heurístico se obtuvieron con los reportes generados por cada una de las herramientas.

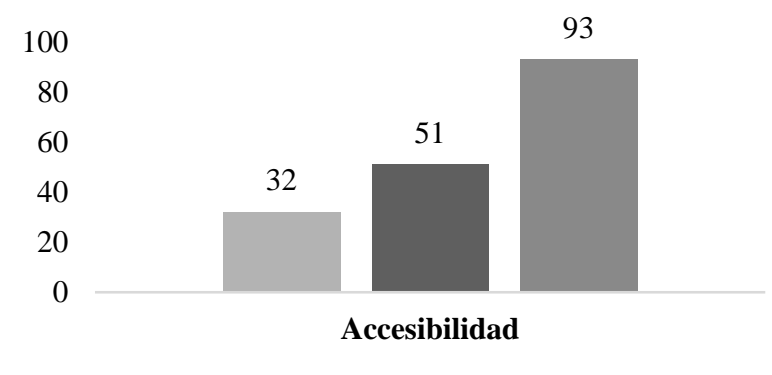

Funtional Accessibility Examinator $\quad$ Lighthouse

Gráfico 7 Evaluación de la Accesibilidad de acuerdo con las herramientas heurísticas empleadas

El Gráfico 7 muestra el porcentaje de cumplimiento de los criterios de Accesibilidad de cada herramienta de evaluación.
De acuerdo con la herramienta Funtional Accessibility el portal cumple con un $32 \%$, un $51 \%$ según Examinator y un $93 \%$ de acuerdo con Lighthouse.

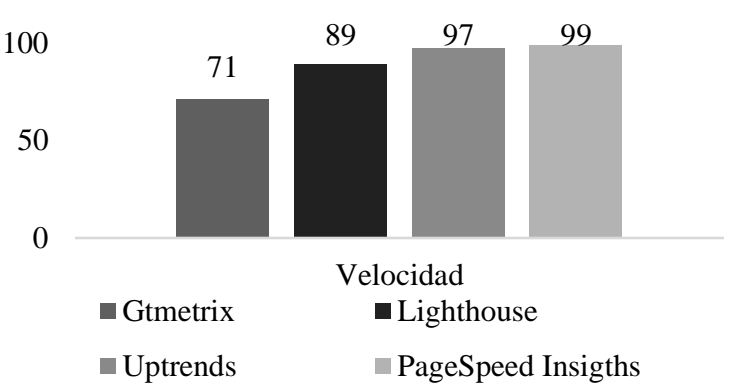

Gráfico 8 Evaluación de la Velocidad de acuerdo con las herramientas heurísticas empleadas

El Gráfico 8 muestra el porcentaje de cumplimiento de los criterios de Velocidad de cada herramienta de evaluación. De acuerdo con la herramienta Gtmetrix el portal cumple con un $71 \%$, un $93 \%$ según Lighthouse, un $97 \%$ de acuerdo con Uptrends y un $99 \%$ para PageSpeed Insigths.

\section{Conclusiones}

La evaluación de usabilidad de la aplicación web SAEFI con la Norma Estándar Internacional ISO 25010 en su primera fase en el método empírico sobre el modelo de calidad de producto, nos arroja una tendencia lineal entre el $60 \%$ y el $80 \%$ en la métrica óptima de la encuesta realizada a los docentes usuarios de SAEFI. Los resultados anteriores proporcionan información para generar acciones de mejora continua en el Sistema. Sin embargo, cabe resaltar que en la subcategoría Operatividad, los indicadores $\mathrm{IO}_{2}$ y $\mathrm{IO}_{3}$ llegaron a ser evaluados hasta con $9.1 \%$ en la métrica 4, es decir el valor cualitativo de Regular, generando una acción correctiva para mejorar los mensajes de error que proporciona el sistema al usuario, con la finalidad de mejorar el control y facilitar su empleo.

Sobre el modelo de calidad de uso, la evaluación de Accesibilidad de la aplicación web SAEFI con la ISO 25010 en su segunda fase con el método heurístico nos proporciona una media entre $93 \%$ y $32 \%$, es decir $61 \%$, generando una acción correctiva respecto al acceso a toda la funcionalidad mediante el teclado y alternativas textuales para que el contenido textual y no textual se pueda convertir en otros formatos para personas con debilidad visual.

MEX-ALVAREZ, Diana Concepción, HERNÁNDEZ-CRUZ, Luz María, UC-RIOS, Carlos Eduardo y CAB-CHAN, José Ramón. Análisis de usabilidad web a través de métricas estandarizadas y su aplicación práctica en la plataforma SAEFI. Revista de Tecnologías Computacionales. 2019 
Por su parte la evaluación de la velocidad nos proporcionó parámetros que oscilan del $71 \%$ al $97 \%$ de cumplimiento, concluyendo mejorar la velocidad de la aplicación, minimizando el número de peticiones y el peso de sus elementos.

\section{Trabajos futuros}

Es conveniente realizar un estudio sobre las diferentes herramientas empleadas para la evaluación de la Accesibilidad, ya que Lighthouse, al ser una extensión de Chrome, tiene sus propios criterios y no todos se apegan totalmente a la norma; así como las herramientas Examinator y Funtional Accessibility Evaluator 2.0 que ambas consideran todas las directrices de la Web Content Accessibility Guidelines (WCAG 2.0)

Respecto a las herramientas de la evaluación de la velocidad, GTmetrix evalúa el número de peticiones que realiza un usuario para poder por visitar por completo la aplicación, y por su parte Lighthouse y Uptrends evalúan el tiempo de carga de cada elemento de la página hasta que están todas disponibles. Por otro lado, es conveniente verificar las "buenas prácticas" que PageSpeed Insigths verifican que se cumplan sin verificar el número de peticiones ni la carga de los elementos.

\section{Agradecimiento}

Agradecemos a la Facultad de Ingeniería de la Universidad Autónoma de Campeche, por las facilidades otorgadas para la realización de esta investigación.

\section{Referencias}

Arroyo Paz, Antonio. (2018) Estudio Comparativo de Plataformas Middleware OpenSource de Internet de las cosas (IoT) Basado en Métricas Cuantitativas y Cualitativas. (Tesis de Magister) Universidad Andina Néstor Cáceres Velásquez.Juliaca Perú.

Barrios García, J. A., Sahagun Montoya, L. A., Bañuelos Rodarte, M., \& Moreira Galvan, J. C. (2018). Implementación de la norma ISO/IEC 29110 de Ingeniería de Software en Instituciones Académicas. Revista de Tecnología y Educación, 1-6.

Benavidez, C. (Abril de 2015). examinator. Obtenido de http://examinator.ws/
British Standards Institution. (2011). Systems and Software Engineering - Systems and Software Quality Requirements and Evaluation (SQuaRE) - System and Software Quality Models. Preston (Reino Unido) : British Standards Intitution .

Enriquez, J. G., \& Casas, S. I. (2013). Usabilidad en aplicaciones móviles. Informes Científicos Técnicos - UNPA, 25-47.

Febrero, F., Calero, C., \& Moraga, Á. M. (2016). Software reliability modeling based on ISO/IEC SQuaRE. Information and Software Technology, 18-29.

França, J., \& Soares, M. (2015). SOAQM: Quality Model for SOA Application based on ISO 25010. International Conference on Enterprise Information Systems, 60-70.

Fundación del Seminario Iberoamericano sobre Discapacidad y Accesibilidad en la Red. (15 de Diciembre de 2009). Pautas de Accesibilidad para el Contenido Web (WCAG) 2.0. Obtenido de

http://www.sidar.org/traducciones/wcag20/es/ Google. (Julio de 2018). PageSpeed Insights. Obtenido de https://developers.google.com/speed/pagespeed /insights/

Google. (14 de Mayo de 2019). Auditar apps web con Lighthouse - Tools for Web Developers - Google Developers. Obtenido de https://developers.google.com/web/tools/lighth ouse/

GT.net. (2019). GTmetrix. Obtenido de https:/gtmetrix.com/

Jiménez, Nelly Josefina \& Lugones, Manuel Ernesto. (2015). Evaluación de la Usabilidad de un Sitio Oficial de la Administración Pública. (Trabajo Final de Graduación de Licenciatura en Sistemas Computacionales). Universidad Nacional de Santiado del Estero.

Mascheroni, M. A., Greiner, C. L., Petris, R. H., Dapozo, G. N., \& Estayno, M. G. (2012). Calidad de Software e Ingeniería. XIV Workshop de Investigadores en Ciencias de la Computación, 656-659. 
Mex Alvarez, D. C., Escamilla de la Cruz, M., Estrada Segovia, G. M., Hernández Cruz, L. M., \& Ortiz Cuevas, N. G. (2019). Beneficios de la Implementación de los Estándares de Calidad para la Ingeniería de Software. Revista Ciencia, Ingeniería y Desarrollo Tec Lerdo, 1-7.

Rubin, J., \& Dana, C. (2008). Handbook of Usability Testing: How to plan, Desing, and Conduct Effective Tests. Indianápolis (Indiana): Wiley Publishing, Inc.

University of Illinois. (2019). Run FAE: Functional Accessibility Evaluator 2.0. Obtenido de https://fae.disability.illinois.edu/anonymous/?A nonymous\%20Report=/

UPTRENDS. (2019). Website Speed Test Uptrends. Obtenido de https://www.uptrends.com/tools/website-speedtest

Xool Clavel, J. I., Buenfil Paredes, H. F., \& Dzul Canche, M. E. (2018). Desarrollo e implementación de un sistema web para el proceso de estadía. Revista de Tecnologías de la Información y Comunicaciones, 8-19. 